\title{
Identification Of The Dominant Noise Source Using Pass-By Time History
}

\author{
WU Di ${ }^{1, a}$ \\ ${ }^{1}$ Dalian Scientific Test and Control Technology Institute, Dalian, China \\ awdi0225@163.com
}

Keywords: Noise Source Identification, Fault Diagnosis, Noise Control

Abstract. Identification of the dominant source could provide instructive guidance for noise mitigation. In this paper, the relation between aerodynamic noise and rolling noise was quantitatively analyzed based on the characteristics of high speed train's pass-by noise. The results indicate that at the speed of $300 \mathrm{~km} / \mathrm{h}$, rolling noise is $5.2 \mathrm{~dB}$ higher than aerodynamic noise, and aerodynamic noise becomes predominant as the speed increasing to $380 \mathrm{~km} / \mathrm{h}$.

\section{Introduction}

High-speed trains now have increasingly become an important means of public transportation, while many rural and metropolitan areas are experiencing the increasing problem of railway noise. Therefore, the prediction and mitigation of railway noise draw more and more attention in the world. Usually, the noise emitted by a single train can be represented by the time history of the sound pressure level. Since the pass-by time history provides large amount of information about railway noise, such studies would help create a better understanding of the railway noise.

Rolling noise and aerodynamic noise are the main noise sources for high-speed trains. Beamforming algorithm has been proposed in order to evaluate contributions of different sources from train pass-by noise $[1,2]$. However, this method has some limitations: the microphone arrays require complicated equipment and high testing cost, additively, this method has low resolution on low-frequency noise. In this paper, an analysis method was proposed for quantification of contributions of the component sources based on pass-by noise curve of high-speed train. The relation between rolling noise and aerodynamic noise during operation of China's high-speed train was obtained. During measurement, only one microphone is required, leading to substantial testing cost reduction and efficiency improvement.

\section{Calculation Method}

When a moving source passes through a fixed observation point at constant speed, the slope of the noise curve on both curve sides are determined by velocity and directivity of the source. When multiple sources with different directivities pass through the observation point simultaneously, the slope of the noise curve is determined by relative strengths of the component sources.

Noise source of a train is usually modelled as a line source or a string of point sources, despite of the variance in selecting parameters such as sound source directivity, height, intensity and attenuation factor of sound propagation. The sound pressure due to the unit length line source may be written as:

$$
p^{2}=\frac{\rho_{0} \sigma_{0} W}{4 \pi r^{2}} D^{2}(\varphi)
$$

where $W$ denotes the sound power of the unit length, $\varphi$ is the horizontal angle , $D(\varphi)$ is the horizontal directivity indices, $\rho_{0} c_{0}$ is the characteristic impedance of air, and $r$ is the source-receiver distance. 
For high-speed trains, the most important noise sources are rolling noise and aerodynamic noise [2]. According to Zhang and TAM's studies [3, 4], rolling noise can be modelled as a dipole, and the directivity of aerodynamic noise can be referred to as:

$$
\Delta L^{A}(\varphi)=10 * \lg \left(0.01+0.5 \cos (\varphi)+0.49 \cos ^{2}(\varphi)\right)
$$

The total pass-by noise of high-speed train could be considered as the superposition of rolling noise and aerodynamic noise. The sound power ratio of aerodynamic noise to rolling noise was denoted as:

$$
k=\frac{w_{A}}{w_{R}}
$$

where $\mathrm{W}_{\mathrm{A}}$ denotes the sound power of the aerodynamic noise, and $\mathrm{W}_{\mathrm{R}}$ denotes the sound power of the rolling noise. The method proposed in [5] was used to calculate the pass-by noise time history under the combined effects of the two noise sources.

The value of $\mathrm{k}$ could be obtained by iteration when the model-predicting curve is the closest match to the measured one. The difference between aerodynamic noise and rolling noise can be determined as follows:

$$
\Delta \mathrm{SPL}=\mathrm{SPL}_{\mathrm{A}}-\mathrm{SPL} \mathrm{R}_{\mathrm{R}}=10 \log k
$$

where $\mathrm{SPL}_{\mathrm{A}}$ denotes sound pressure level due to the aerodynamic noise, and SPL $\mathrm{R}_{\mathrm{R}}$ denotes sound pressure level due to the rolling noise.

\section{Results and discussions}

Based on this method, the analysis was taken on total noise emission of CRH-380AL(a Chinese electric high-speed train that is developed by China South Locomotive \& Rolling Stock Co., Ltd.) running at the speed of $300 \mathrm{~km} / \mathrm{h}$ and $380 \mathrm{~km} / \mathrm{h}$. The measurements were carried out according to the standard of ISO 3095-2005. At the location where the measurements were made, the terrain was mostly flat. There were no trees or other reflecting obstacles. The track was straight and in good condition, and there was no interference from other acoustic sources. The microphone was placed at a distance (from the track's center) of $25 \mathrm{~m}$ and at a height (from the track plane) of $3.5 \mathrm{~m}$.

At $300 \mathrm{~km} / \mathrm{h}$, the value of $\mathrm{k}$ is 0.3 meaning that rolling noise, $5.2 \mathrm{~dB}$ higher than aerodynamic noise, is predominant under this situation. This result is basically consistent with that derived in [6], while different analysis method is adopted. In [6], the author suggests that the aerodynamic noise is not important below $300 \mathrm{~km} / \mathrm{h}$. The methods proposed in these two papers can by mutually verified. The comparison of predicted curve and measured curve was shown in Fig.1, it can be observed that the two curves are very close with an average error of $1.2 \mathrm{~dB}$. This result suggests that it is reasonable to consider the pass-by noise as superposition of rolling noise and aerodynamic noise while other noises are negligible. 


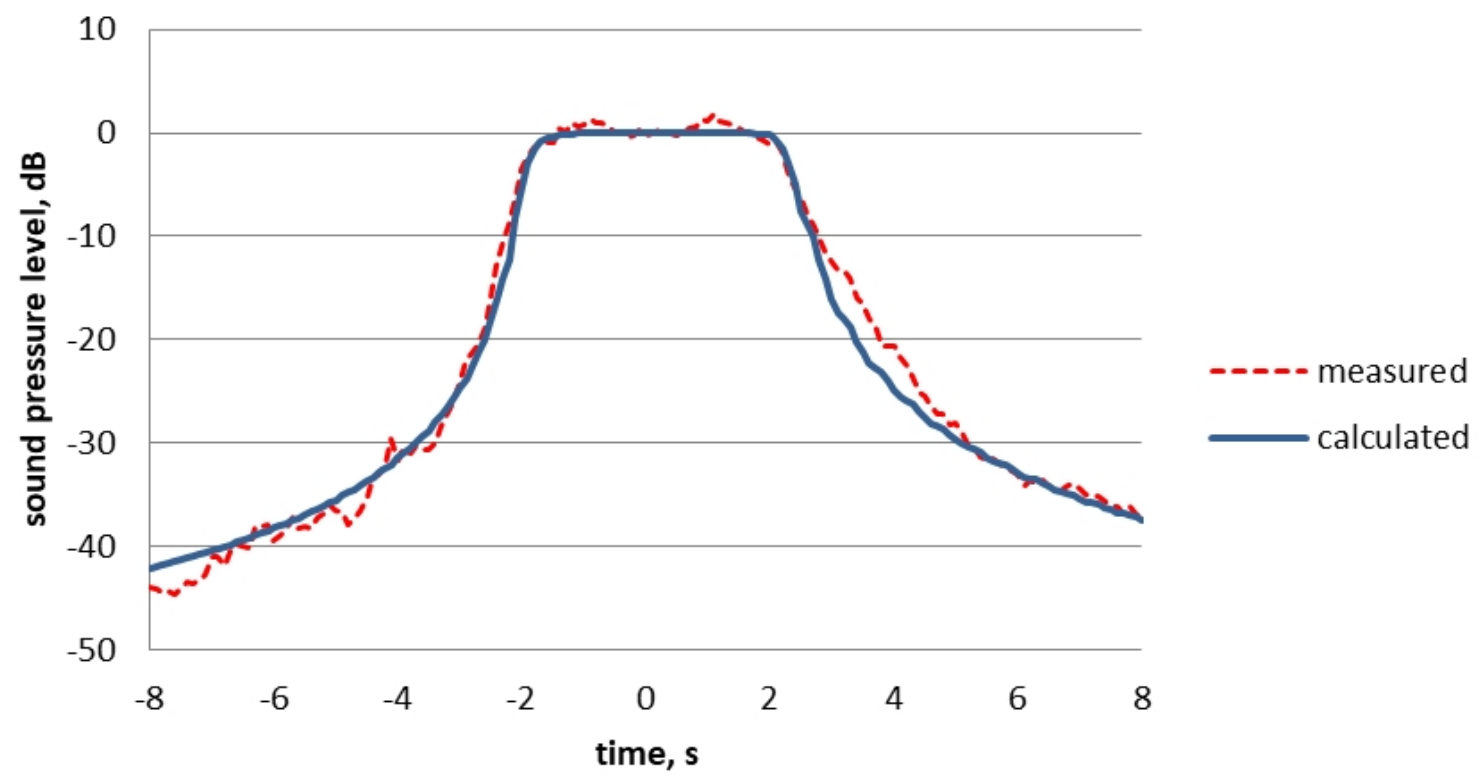

Fig. 1 Calculated time history of sound pressure level compared with measured data (velocity, v =300 km/h)

At the speed of $380 \mathrm{~km} / \mathrm{h}$, aerodynamic noise, $11 \mathrm{~dB}$ higher than rolling noise (with $\mathrm{k}$ equals to 14 ), becomes the dominant of pass-by noise.

Based on this analysis, the transition speed should be above $300 \mathrm{~km} / \mathrm{h}$ which is higher than the result from [1]. The difference may be due to the relative poor wheel/rail processing technic of Chinese high speed train.

\section{Application}

The method proposed in this paper can be used for the identification of noise sources and also for fault diagnosis. By using a microphone placed beside the railway to record the pass-by time history, the train's condition can be judged by analyzing the proportion relation between the wheel rail noise and the aerodynamic noise. According to the previous analysis, even the wheel rail noise is much lower than the aerodynamic noise, the abnormal of rolling noise can still be identified according to the change of the slope of the time history.

\section{Conclusion}

In this paper, a quantification method of high-speed train noise sources was proposed based on pass-by noise curves. For CRH380AL, at the speed of $300 \mathrm{~km} / \mathrm{h}$, rolling noise is $5.2 \mathrm{~dB}$ higher than aerodynamic noise; as the speed increasing to $380 \mathrm{~km} / \mathrm{h}$, aerodynamic becomes predominant.

\section{Acknowledgements}

The author is grateful to YAN Shuang for the help she provided during the study.

\section{References}

[1]. Mellet, C., et al., High speed train noise emission: Latest investigation of the aerodynamic/rolling noise contribution. JOURNAL OF SOUND AND VIBRATION, 2006. 293(3-5SI): p. 535-546.

[2]. Thompson, D.: Railway Noise and Vibration. Elsevier, Oxford. 2009 
[3]. Zhang, X.T. and H.G. Jonasson, Directivity of railway noise sources. JOURNAL OF SOUND AND VIBRATION, 2006. 293(3-5SI): p. 995-1006.

[4]. TAM, C., INTENSITY, SPECTRUM, AND DIRECTIVITY OF TURBULENT BOUNDARY-LAYER NOISE. JOURNAL OF THE ACOUSTICAL SOCIETY OF AMERICA, 1975. 57(1): p. 25-34.

[5]. Wu, D. and J. Ge, The Asymmetry of the Time History of the High-Speed Train Pass-By Noise. ACOUSTICS AUSTRALIA, 2015. 43(2): p. 169-173.

[6]. Zhang, X., Empirical Modeling of Railway Aerodynamic Noise Using One Microphone Pass-By Recording, in Notes on Numerical Fluid Mechanics and Multidisciplinary Design, J. Nielsen, et al., J. Nielsen, et al. Editors. 2015, SPRINGER-VERLAG BERLIN: BERLIN. p. 125-131. 